\title{
La pratique philosophique avec les enfants, un dispositif en résonance avec le paradigme de l'éducation complexe
}

The practice of philosophy with children, a pedagogical setting resonating with the paradigm of the complex education

Johanna Hawken

\section{(2) OpenEdition}

Journals

Édition électronique

URL : http://journals.openedition.org/trema/6088

DOI : $10.4000 /$ trema.6088

ISSN : 2107-0997

Éditeur

Faculté d'Éducation de l'université de Montpellier

Référence électronique

Johanna Hawken, «La pratique philosophique avec les enfants, un dispositif en résonance avec le paradigme de l'éducation complexe », Tréma [En ligne], 54 | 2020, mis en ligne le 01 décembre 2020 consulté le 14 décembre 2020. URL : http://journals.openedition.org/trema/6088 ; DOI : https:// doi.org/10.4000/trema.6088

Ce document a été généré automatiquement le 14 décembre 2020.

Trema 


\section{La pratique philosophique avec les enfants, un dispositif en résonance avec le paradigme de l'éducation complexe}

The practice of philosophy with children, a pedagogical setting resonating with the paradigm of the complex education

Johanna Hawken

\section{Introduction}

1 Il semble que le monde éducatif soit interpelé par de nouvelles problématiques spécifiques à la société contemporaine: il n'est pas anodin que les travaux d'Edgar Morin et ceux des philosophes pour enfants se soient élaborés simultanément, depuis les années 1970. Les auteurs mettent en valeur la nécessité d'une formation de la pensée.

2 La faculté de penser se trouve au coeur du nouveau paradigme de l'éducation complexe proposée par Edgar Morin (1999a). En effet, la complexité du monde et de la condition humaine exige d'instituer une éducation de la pensée chez l'enfant, afin que sa cognition soit le siège d'une appréhension clairvoyante et sereine de la société humaine. Elle se trouve également au centre de la vision de Matthew Lipman, père fondateur de la Philosophie Pour Enfants (PPE).

3 La pratique de la philosophie avec les enfants, âgés de 4 à 17 ans, constitue une innovation pédagogique qui résonne fortement des travaux d'Edgar Morin, si bien qu'il en est devenu l'un de ses plus ardents défenseurs ${ }^{1}$. Dès son apparition, dans les années 1960, la philosophie pour enfants a pour ambition d'outiller intellectuellement les futurs citoyens (Lipman, Oscanyan, Sharp, 1990): elle considère que la faculté de pensée pourrait être le socle d'une permanence face aux changements, qu'elle pourrait 
créer des compétences cognitives solides et pérennes face à la variété et à la diversité des situations, des contextes, des informations et des savoirs.

4 Il convient d'établir que la philosophie pour enfants se distingue bien sûr, des formes traditionnelles de l'enseignement philosophique (études de texte, commentaires, dissertations). Pour autant, elle revendique l'instauration d'un modèle didactique constituant un véritable apprentissage du philosopher. Au-delà des différents courants qui la constituent, il est possible de dégager une certaine vision commune de la philosophie, telle qu'elle est ciblée ici. Elle se définit comme une pratique de la réflexion sur l'expérience humaine visant l'exploration de concepts et questions universels, au sein d'une discussion collective mobilisant certaines compétences de pensée. Il s'agit, au fond, de renouer avec une vision ancestrale de la philosophie, et d'offrir aux élèves un moment dédié à la découverte des idées qui sommeillent en eux, à la croissance de leur faculté de pensée de leurs idées et à l'exploration de la pluralité des idées (vraies, fausses, authentiques, reçues, conscientes, inconscientes) qui circulent dans la société. Vouloir intégrer la philosophie dans leur éducation, c'est tenter de renouer et de renforcer les liens entre les enfants, les idées et le monde, et œuvrer ainsi pour que cette relation triangulaire soit plus libre et consciente.

«Les idées existent par et pour l'homme, mais l'homme existe aussi par ou pour les idées. Nous ne pouvons bien nous en servir que si nous savons aussi les servir. $\mathrm{Ne}$ faut-il pas prendre conscience de nos possessions pour pouvoir dialoguer avec nos idées, les contrôler autant qu'elles nous contrôlent (...) ?» (Morin, 1999b : 29).

Animée, aussi, par la volonté d'initier une « réforme de la pensée » (Morin, 1999a), la philosophie pour enfants pourrait donc être l'une des pratiques pédagogiques qui pourraient participer à la construction d'une éducation complexe. Cette intrication entre le projet d'Edgar Morin et celui de la philosophie pour enfants se joue notamment à trois endroits, que je souhaiterais analyser successivement au sein de mon article : par son objet d'étude, par sa méthode et par sa visée.

Par son objet: La condition humaine, notamment ses dimensions épistémologiques et éthiques.

Par sa méthode : La pratique du dialogue collectif, intersubjectif et pluraliste.

Par sa visée : La formation de la pensée multidimensionnelle.

7 Pour explorer ces trois axes, je m'appuierai, d'une part, sur la littérature constituée ces dernières décennies sur la philosophie pour enfants et, d'autre part, sur ma pratique expérimentale, au sein d'une recherche-action que je mène depuis 2011 en Seine SaintDenis (d'abord au sein d'une recherche doctorale, puis au sein de la Maison de la Philo, qui a été créée à sa suite). La Maison de la Philo, créée en 2014, est une structure municipale dédiée aux pratiques philosophiques, en partenariat avec toutes les structures éducatives, sociales et culturelles de la ville (écoles, collèges, centres de loisir, cinéma, médiathèque, centres sociaux etc.). Celle-ci me permet d'animer des ateliers philosophiques dans les cadres scolaires, périscolaires et extrascolaires, et vise à démocratiser ainsi la philosophie auprès de tous les habitants (enfants, adolescents, adultes, retraités). Dans ces divers cadres, l'animateur-philosophe prend en charge l'atelier philosophique et le partenaire éducatif assure la tenue du cadre et une écoute attentive, mais n'intervient pas dans la discussion.

De multiples méthodes se sont développées depuis les années 1970 (la Communauté de Recherche Philosophique, les Ateliers ARCH, la Discussion à Visée Démocratique et 
Philosophique), mais mon expérience m'a poussée à constituer une méthode spécifique en me nourrissant fortement de ces travaux. J'applique dans mes ateliers une méthode que j'ai constituée au cours de ma recherche doctorale, en articulant les travaux de mes aînés, et en les complétant de certains outils ou techniques pédagogiques qui m'ont paru pertinents et nécessaires. Elle se nomme «Communauté d'Éclosion Conceptuelle » et, comme son nom l'indique, elle fonde la réflexion philosophique sur l'exploration d'un concept central dans la vie des enfants et adolescents : liberté, bonheur, amour, vie, justice, bien, mal, amitié, émotion, différence, identité, humain, animal. Par ce processus de conceptualisation, elle vise à éclore une problématique, dans la continuité de la définition collective. Voici son déroulement-type, tel qu'il est applicable avec des élèves de 6 à 16 ans (ma recherche étant ciblée sur les cycles 2,3,4) :

1. Rituel d'entrée (3') : Présentation de la philosophie, rappel des règles et du droit de chacun à s'exprimer et de la capacité de chacun à s'exprimer.

2. Conceptualisation (10-15'): Recherche de sens autour du concept du jour «Qu'est-ce que ... ?» : minute de réflexion puis tour de parole donnée à tous les participants, pour tenter de définir le concept. Ce moment est une prise de la méthode ARCH (Lévine : 2008).

3. Synthèse (2') de la conceptualisation et présentation d'une première problématique en ayant émergé.

4. Échange collectif (10-20') : à partir de la problématique dégagée, recherche de sens et utilisation des compétences de pensée sous la guidance du facilitateur.

5. Synthèse (2') et émergence d'une deuxième problématique: Tour de parole pour tous les participants.

6. Présentation d'un support inducteur (5') choisi en fonction du concept du jour (album jeunesse, court-métrage, œuvre d'art, etc.), suivie d'une question philosophique, selon les recommandations méthodologiques proposées par Edwige Chirouter ${ }^{2}$ (Chirouter : 2007).

7. Échange collectif (10-20') : Réflexion à partir du support inducteur et de sa question. À partir de la problématique dégagée, recherche de sens et utilisation des compétences de pensée.

8. Rituel de fin (2') : Synthèse. Félicitations et présentation d'axes de progression.

9 Tout au long de ce processus, la recherche philosophique des élèves se développe grâce à la mobilisation de dix compétences de pensée, nécessaires pour que l'atelier ait une teneur philosophique et se distingue des conversations et bavardages ordinaires. Les philosophes pour enfants débattent sur le nombre et la nature des compétences nécessaires au philosopher, oscillant entre trois (Tozzi, 2001) et soixante-douze (Lipman, 2008; Sasseville, 2008). Mon expérience m'a montrée que dix compétences intellectuelles sont absolument indispensables à la mise en place d'une discussion philosophique, en tant qu'elles sont utilisées soit par le facilitateur, soit par les participants : questionner, créer des idées, conceptualiser, argumenter, problématiser, illustrer, distinguer, raisonner, critiquer et dialoguer. Celles-ci permettent de partir des premières idées et pensées des élèves et de les approfondir, de les développer, en utilisant ces diverses compétences. Nous analyserons leur fonction dans le détail, mais pouvons d'ores et déjà établir qu'elles sont essentielles. Ce sont elles qui renforcent et complexifient la relation des élèves aux idées et au monde. 


\section{La discussion philosophique, pratique éducative propice à la prise de conscience des enjeux de la condition humaine.}

\section{La place de la réflexion sur la condition humaine.}

10 La philosophie pour enfants s'inscrit dans un courant philosophique spécifique, dont la tâche principale est l'exercice d'une réflexion sur l'existence, l'expérience et le vécu philosophique. Loin d'adhérer aux écoles philosophiques rationalistes, systémiques ou idéalistes, elle ne se définit pas comme un enseignement de l'Histoire de la pensée, une étude des systèmes théoriques d'élaboration de concepts abstraits. Le courant "Philosophie Pour Enfants» (PPE) est porté par une vision existentielle et expérientielle de la pratique philosophique, si bien qu'elle se consacre tout entière à initier les enfants et adolescents à la réflexion sur la condition humaine. Les philosophes pour enfants considèrent, contre la tradition philosophique de l'enfant aveugle, innocent, inconscient, enclin à l'erreur et aux préjugés (Descartes, 1996), que chaque individu acquiert dès le plus jeune âge une expérience signifiante de l'existence. Bien qu'au cours la jeunesse, il ne possède pas une somme conséquente de vécus, il éprouve certaines questions vives de l'existence, dans son rapport à lui, aux autres, à la société : Peut-on être libre dans la contrainte? Les émotions sont-elles plus fortes que nous? Pourquoi les humains se disputent sur le dieu ou les dieux? Comme l'attestent ces questionnements, l'enfant commence à percevoir les problématiques propres à la condition humaine. Il est donc fécond de s'appuyer sur cette curiosité philosophique pour initier une prise de conscience des enjeux inhérents au fait d'être humain. «L'éducation devrait comporter un enseignement premier et universel portant sur la condition » (Morin, 1999b : 49).

11 Ce mouvement est essentiel et résonne avec le modèle de l'éducation complexe : en effet, Edgar Morin déplore l'hyperspécialisation des champs de connaissance, la fragmentation des savoirs dans les diverses disciplines, ainsi que leur disjonction (1999b : 42). Le modèle actuel engage chaque domaine de connaissance à analyser des problèmes particuliers, si bien que l'on perd de vue les problèmes globaux, qui sont pourtant cruciaux. La philosophie peut redevenir ce lieu dédié à l'analyse de la globalité : en effet, elle permet d'étudier un problème général dans ses divers contextes en se nourrissant des apports des sciences.

12 Ainsi, les ateliers philosophiques sont généralement construits autour de l'exploration d'un concept, accompagné d'une ou plusieurs problématiques philosophiques centrales pour la société humaine. Par exemple, si le concept choisi est "La Liberté », il est possible que la réflexion soit dédiée aux questions suivantes : "Sommes-nous libres de faire ce que l'on veut?", "Quelles sont les limites de la liberté ? ", "Quelle est la différence entre être libre et se sentir libre?", "Avons-nous vraiment la liberté de choisir?", "Assouvir une envie, est-ce toujours se rendre libre?». Les ateliers philosophiques visent à explorer des concepts et problèmes qui sont à la fois centraux dans l'Histoire de la philosophie et cruciaux dans l'expérience de l'enfant. Ainsi, ils constituent une opportunité importante pour œuvrer en vue d'une prise de conscience de ces questions. 
13 En outre, les questions philosophiques ont pour caractéristique de révéler simultanément l'unité et la diversité de la communauté humaine. En effet, elles sont à la fois fédératrices et polémiques : certaines grandes questions préoccupent tous les humains, dès le plus jeune âge, mais chacun peut y apporter une réponse différente. Les enfants et adolescents le ressentent fortement dans la pratique philosophique : leurs témoignages montrent qu'ils sont étonnés à la fois de la similarité de leurs questionnements et de la diversité des réponses possibles. Les problèmes philosophiques permettent ainsi de prendre conscience de l'« Unitas multiplex » chère à Edgar Morin : souhaitant mettre en lumière l'unité et la diversité humaine, il déclare que « l'éducation devra veiller à ce que l'idée d'unité de l'espèce humaine n'efface pas celle de diversité et que celle de diversité n'efface pas celle de l'unité » (Morin, 1999b : 58). L'exploration des questions universelles et polémiques de la philosophie permet de faire éprouver cette coexistence entre unité et diversité dans l'espèce humaine.

14 Nous présenterons quelques exemples importants pour la philosophie éthique et épistémologique, en tant qu'ils constituent deux domaines particulièrement importants tant pour la philosophie pour enfants que pour l'éducation à la pensée complexe.

\section{La réflexion philosophique sur les questionnements éthiques de la condition humaine}

15 Les ateliers philosophiques visent à explorer des concepts et problèmes qui sont à la fois centraux dans l'Histoire de la philosophie et cruciaux dans l'expérience de l'enfant. La condition enfantine rencontre déjà certains grands problèmes éthiques. En ce qui concerne les ateliers dédiés à la philosophie éthique, voici les concepts et problèmes qui sont pertinents à la fois dans l'histoire de la pensée et dans la vie de l'enfant :

- Le bien : Comment savoir ce qui est bon? Qu'est-ce qu'une bonne personne? Une bonne intention débouche-t-elle toujours sur une bonne action?

- Le mal : Qu'est-ce qui est mal ? A-t-on toujours conscience du mal que l'on fait? Quelle différence entre faire du mal et vouloir faire du mal?

- Le juste / l'injuste: Qu'est-ce qu'une injustice? Le sentiment d'injustice est-il naturel ? Faut-il combattre l'injustice? Comment faire ce qui est juste?

-L'égalité : Sommes-nous tous égaux ? Que ressent-on face à une inégalité ?

- Les lois : Les humains ont-ils besoin de lois ? Qu'est-ce qu'une loi juste?

- Les règles : Les règles sont-elles utiles ? Une même règle vaut-elle pour tous?

- L'obéissance : Faut-il toujours obéir ? Peut-on choisir librement d'obéir?

- Le pouvoir : A-t-on besoin de chefs? Les chefs ont-ils toujours raison? Y a-t-il une bonne façon d'exercer un pouvoir? Quelle différence entre la force et la pouvoir?

-Autrui : Les autres sont-ils mes semblables ? Est-il juste de rejeter quelqu'un de différent?

- La nature: Les humains sont-ils les maîtres de la nature? Doit-on prendre soin de la nature ? Les humains ont-ils des responsabilités face à la nature?

L'exploration de ces concepts et questions éthiques permet de mener un travail de conscientisation et de sensibilisation: conscients et attentifs aux questions éthiques partagées, les enfants pourraient accroître leur capacité à les saisir. Si l'on considère que la racine d'une démarche éthique consiste dans le fait même d'y porter son attention, alors le fait, pour les enfants, de prendre le temps d'y penser, collectivement, constitue - en soi - une expérience éducative porteuse d'une teneur éthique. Il s'agit 
dans l'activité philosophique, de les rendre sensible à l'importance, à la complexité, et à la subtilité des questions éthiques.

Nous pouvons prendre pour exemple un atelier de philosophie effectué durant le temps méridien, dans une école élémentaire de Romainville, avec six enfants âgés de huit ans. Cet atelier portait sur la question du Bien et du Mal, à partir de la lecture d'un album fort connu : Les trois Brigands (Ungerer, 1961).

"[Facilitatrice]: Aujourd'hui, on va discuter du Bien et du Mal. Le thème aujourd'hui, c'est donc «Le Bien et le Mal»: ce sont des mots philosophiques, on a vu la dernière fois que les mots philosophiques ont beaucoup de sens. On va réfléchir à partir d'une histoire qui est très connue, mais je ne pense pas que vous avez déjà philosophé sur cette histoire. Donc là, le but, c'est d'écouter l'histoire, de se laisser aller, mais par contre il faut penser, penser, penser. Dans cette histoire, il y a plein d'idées et de questions sur le bien et le mal. [Lecture de l'album] Les brigands, est-ce qu'ils font le bien ou est-ce qu'ils font le mal?

- [Enfant 1]: Je crois qu'ils font les deux, parce qu'au début ils sont méchants et quand ils ont rencontré Tiffany, et ils ont commencé à prendre soin des orphelins.

- [Enfant 2] : Ils font le bien, tout le temps. Parce que même au début, et bah c'est pour eux, voler c'est leur métier, c'est normal.

- [Enfant 3] : Moi je dis que c'est les deux parce qu'au début ils aiment embêter les gens, ils s'amusent, ils font juste ça parce qu'ils s'ennuient et ensuite ils trouvent Tiffany, qui leur a dit quoi faire avec l'or et ils ont fait un château pour les orphelins. Et après ils sont devenus gentils

- [Enfant 4] : Ils sont gentils, avant qu'ils volaient, mais après ils faisaient un château et des manteaux pour les enfants

- [Enfant 5] : Oui parce qu'ils aiment bien les enfants

- [Enfant 6] Les enfants ils étaient pauvres, et ils leur ont acheté un château

- [Enfant 1] : De style ils sont plutôt méchants mais au fond d'eux, ils sont plutôt gentils

- En fait, au début, ils étaient méchants parce qu'ils prenaient l'argent des gens mais quand ils ont rencontré Tiffany, ils ont vu la valeur d'un enfant, ils se sont dit «la pauvre elle est toute seule, on devrait lui donner de quoi vivre. »

- [Enfant 3] : Moi je dis les deux parce qu'au début ils font du mal aux gens et ensuite ils deviennent un peu plus gentils.

- [Enfant 5] : Ils sont gentils parce que comme Tiffany elle a trouvé de l'or, bah ils se sont dit qu'ils pouvaient prendre des enfants abandonnés

- [Enfant 6] : Ils sont méchants, ils sont beaucoup gentils et un peu méchant. Parce que avec les humains, ils sont méchants, mais avec les enfants ils sont pas méchants.

- Mais les enfants c'est des humains

- [Enfant 3] : Moi je pense qu'ils sont gentils pendant tout le livre, c'est juste qu'ils volaient des gens pour avoir de la richesse.

- [Enfant 2] : Moi je pense qu'ils ont pas changé : au début ils étaient gentils mais juste ils volaient les gens, c'est tout

- [Facilitatrice] : C'est intéressant parce que je vous ai demandé si les trois brigands faisaient du bien ou du mal et vous m'avez répondu : au début ils sont méchants et ensuite ils sont gentils. Alors, vous êtes passés de faire le mal à la méchanceté, et de "faire le bien" à la gentillesse. Pourquoi vous êtes passés de l'un à l'autre?

- [Enfant 4] : Parce qu'être gentil c'est faire le bien.

- [Enfant 5] : Et être méchant, c'est faire le mal.

- [Enfant 2] : Ils sont les deux parce qu'ils volent de l'argent et ils sauvent des enfants. 
- [Enfant 3] : Mais ils font pas le bien parce qu'ils auraient dû rendre l'argent aux gens.

- [Enfant 3] : Moi je pense qu'en fait, au début ils avaient un peu de gentillesse dans eux mais ils le développaient pas, c'est dès que Tiffany elle est venue, ils ont compris qu'il fallait le développer : ça on le sait pas mais c'est ce qu'on imagine.

- [Facilitatrice] : Alors on va revenir sur le début. Ils dévalisent, ils volent, ils font peur : qu'est-ce qu'ils ressentent quand ils font tout cela?

- [Enfant 6] : Ils doivent ressentir le mal : parce que peut-être dans leur peau il y a de la gentillesse. Ils avaient mal à l'intérieur.

- [Facilitatrice] Qu'est-ce que vous en pensez?

- [Enfant 2] : Peut-être que c'est leur manière pour être gentil.

- [Facilitatrice] Pourquoi ils volent tout ce qu'ils volent?

- [Enfant 6] : Moi je pense qu'ils font ça parce que dans leur grotte et au début il y avait pas grand chose et ça leur aide et ils attendaient que quelqu'un vienne pour qu'ils puissent l'aider.

- [Enfant 1] Je pense que c'était juste pour s'occuper, c'est leur métier, comme j'ai dit.

- [Enfant 6] : Ils se sentent tristes parce qu'ils veulent pas faire du mal

- [Enfant 3] : Si ils veulent faire du mal !! ils s'amusent, je crois qu'ils sourient !

- [Facilitatrice] Donc tu penses que ça leur fait plaisir? Vous êtes d'accord?

- [Enfant 5] : Moi je pense qu'ils s'amusaient parce que si ils s'amusaient pas, ils auraient arrêté parce qu'ils se seraient rendus compte que tout le monde les fuyait.

- [Facilitatrice]: Et après pourquoi ils ont acheté le château des orphelins?

- [Enfant 3] : Pour faire plaisir à Tiffany! ils ont juste changé de plaisir !

- [Enfant 2] : Avant ça leur faisait plaisir de faire des choses méchantes et après ça leur fait plaisir de faire des choses gentilles.

- [Facilitatrice] : Donc quelque part, ils n'ont pas changé: c'est toujours leur plaisir qui compte?

- [Enfant 6] : Non ils sont gentils, ils veulent faire du bien.

- [Enfant 2] : Même s'ils ont fait une action bien, ils changeront jamais. (...) ${ }^{\circ}$

- [Enfant 5]: Je ne suis pas d'accord, c'est Tiffany qui leur a donné l'intention de le faire le bien

- [Enfant 4]: Eux ils s'en fichent un petit peu, si elle serait plus là Tiffany, ils recontinueraient à voler.

- [Enfant 1] : En fait, moi je pense que c'est Tiffany qui leur donne la gentillesse.

- [Enfant 1] : Mais ils veulent pas leur faire de mal : ils les tuent pas. (...)

- [Enfant 3]: Oui, si on n'avait jamais fait de mauvaise action, la vie ne serait pas marrante.

- [Enfant 1] : Chacun est un peu égoïste dans son corps ! (...)

- [Facilitatrice] Les brigands, pourquoi ils volent?

- [Enfant 6] : Parce qu'ils veulent être riches

- [Enfant 4] : En fait, ils volent pour vivre

- [Enfant 1] : Non, ils ne font rien avec ce qu'ils volent ! ils ont pas besoin de voler ! (...)

- [Enfant 6] : Pour embêter les habitants

- [Enfant 3] : Parce qu'ils s'ennuient

- [Enfant 2] : Parce qu'ils veulent être riches

- [Enfant 5] : Parce qu'ils aiment faire le mal

- [Enfant 3] : Ils aiment embêter les gens

- [Enfant 1] : Ils aiment leur faire peur. (...)

- [Enfant 3] Parce que pour eux, ce qu'ils font, c'est jouer

- [Facilitatrice] Vous dites «ils aiment faire le mal». Dans l'histoire, on a l'impression qu'ils 
volent pour jouer. Ils se déguisent, ils attrapent les voitures. Ils n'ont pas besoin de voler, mais ils aiment ça. Là on arrive à une question très importante : est-ce que le Mal, c'est faire du mal, ou est-ce que c'est aimer faire du mal ? C'est compliqué, 1, 2, 3, Pensez....

- [Enfant 4] : J'ai pas compris.

- [Facilitatrice] Je répète : est-ce que le mal, la méchanceté, c'est faire du mal ? Ou est-ce que c'est aimer faire du mal? Vous voyez la différence : faire le mal, aimer faire le mal.

- [Enfant 4] : C'est aimer faire le mal (...)

- [Enfant 5] : Nous, si on volait, on sera pas tellement content de voler.

- [Facilitatrice] La question du Bien et du Mal, personne n'a la réponse. Donc on revient sur la question : est-ce que le Mal c'est faire le Mal ou est-ce que c'est aimer faire le mal ?

- [Enfant 6]: C'est juste faire le mal.

- [Enfant 3] : Parce qu'en fait, par exemple si on tue quelqu'un, j'aurais fait du mal, mais j'aurais pas aimer tuer.

- [Enfant 4] : Parce que si par exemple, je vole un objet, et bah c'est mal.

- [Facilitatrice] : Alors on va prendre un autre exemple: si on fait du mal mais sans faire exprès. Imaginez vous avez cassé sans faire exprès trois beaux vases de vos parents : est-ce que c'est mal ou pas? Vous avez fait du mal mais sans vouloir faire du mal. Qu'en pensez-vous?

- [Enfant 2] : Ce n'est pas de la méchanceté

- [Enfant 6] : Si on n'a pas fait exprès, on n'est pas méchant

- [Enfant 1] : Si on fait pas exprès de faire du mal, on fait quand même pas du bien. On l'a pas fait avec volonté.

- [Facilitatrice] On ne l'a pas fait avec volonté. On arrive à notre dernière question du jour. Est-ce que le Mal, c'est vouloir faire du Mal ? 1, 2, 3, Pensez! Est-ce que le Mal, c'est faire du mal ou estce que c'est vouloir faire du mal ? Est-ce que c'est casser un vase parce qu'on veut casser un vase ou est-ce que c'est casser un vase sans faire exprès?

- [Enfant 2] : Ce sera pas du mal parce que c'est sans le vouloir.

- [Enfant 5] : C'est vouloir faire du mal.

- [Enfant 1]: Si on fait du mal alors qu'on veut pas le faire, ça veut dire que c'est pas du mal.

- [Facilitatrice] : Donc pour toi, le Mal, c'est vouloir faire du mal.

- [Enfant 6] : Si on fait pas exprès de faire tomber le vase, c'est plus du bien

- [Enfant 2] : On était énervé et donc on a cassé le vase.

- [Facilitatrice] Dans ce cas, tu voulais le casser ou pas?

- [Enfant 4] : Non, ce n'est pas mal.

- [Enfant 3] : Casser sans faire exprès, c'est pas mal. Par exemple on joue et il y a un vase qui tombe par terre, on voulait pas vraiment le faire tomber, donc c'est pas du mal.

- [Facilitatrice] Donc, on va faire la conclusion de l'atelier. Fermez les yeux. Je vais vous dire les trois grandes questions du jour. La première: le Mal, est-ce que c'est faire du mal ou aimer faire du mal ? La deuxième c'était : est-ce que la Méchanceté c'est vouloir faire du mal ou juste faire du mal ? Et la dernière question c'était : est-ce que parfois il faut faire du mal pour faire du bien? Est-ce que tout le monde a compris les trois questions du jour?

- [Tous] : Oui. $»^{3}$

Cet extrait montre que l'exploration collective des concepts de Bien et de Mal amène à une série de réflexions, de problématiques et d'exemples qui étoffent, progressivement, leur appréhension. Les enfants, accompagnés par la facilitatrice, découvrent la complexité de la définition du Mal : le Mal consiste-t-il à faire du mal, à aimer faire du mal, à vouloir faire du mal ? Ces questions amènent une multitude d'hypothèses qui se trouvent nuancées, au fur et à mesure de l'échange collectif. Nous apercevons donc, ici, 
le rôle de la philosophie pour l'acquisition d'une prise de conscience des subtilités inhérentes aux questions éthiques.

\section{La réflexion philosophique sur les questionnements épistémologiques de la condition humaine}

"L'éducation doit donc se vouer à la détection des sources d'erreurs, d'illusions et d'aveuglements » (Morin, 2000 : 19). Edgar Morin en distingue quatre : erreurs mentales (liées aux rêves, aux fantasmes, à l'imaginaire), erreurs intellectuelles (liées à l'enfermement dans les doctrines, théories, idéologies), erreurs de la raison (liées à la rationalisation extrême, aux fausses déductions, aux logiques fallacieuses), aux aveuglements paradigmatiques (liés au déterminisme culturel, au processus de sélection/rejet de concepts prépondérants). La pratique philosophique est l'occasion de découvrir et travailler les mécanismes de l'erreur, à la fois dans sa méthode, et dans ses sujets de réflexion épistémologique. En effet, non seulement elle engage l'enfant à prendre l'habitude de questionner les vérités, de prendre conscience des idées et d'être prudent vis-à-vis des discours, mais en plus, elle met en place des discussions sur les thèmes de l'illusion, de l'apparence, des préjugés, des informations fausses, des rumeurs. Concernant les erreurs mentales, les enfants reçoivent parfois des idéologies et des théories qui leur sont présentées comme les seules possibles. Découvrant, dans la discussion, qu'il existe d'autres points de vue, ils peuvent s'émanciper de cet enfermement. Concernant les erreurs de la raison, la méthode philosophique repose sur l'idée qu'une idée valable ne sera pas que rationnelle : certaines idées seront mises de côté car elles ne sont pas raisonnables. En effet, la question ne sera pas seulement: est-ce que c'est logique et rationnel ? «Mais est-ce que c'est raisonnable? Est-ce qu'on peut vivre ainsi? Est-ce que cette idée est vraie pour les humains?». Concernant les aveuglements paradigmatiques, les élèves sont encouragés à réfléchir à l'échelle de l'humanité, en explorant des questions, des concepts et des idées ayant du sens " pour tous les humains, depuis toujours et partout", comme je leur dis souvent. Ils sont ainsi invités, par la parole mais aussi par des supports présentant d'autres époques et cultures, à prendre du recul vis-à-vis des déterminismes culturels. Bien entendu, on ne peut nier la part culturelle de nos questionnements, idées et concepts, ne serait-ce que dans leur formulation linguistique. Cela dit, il est fécond de tenter d'acquérir une hauteur de vue, afin de saisir, d'une part, les objets philosophiques universels et, d'autre part, certains objets intellectuels culturellement situés.

- Croire/Savoir : Faut-il croire tout ce que l'on voit? Qui croire? Quelle est la différence entre croire et savoir? Peut-on croire quelque chose sans le savoir?

-L'erreur : A-t-on besoin de faire des erreurs? Que nous enseignent nos erreurs? Peut-on comprendre sans se tromper?

-L'illusion : Qu'est-ce qu'une illusion? Peut-on échapper aux illusions ? Les illusions ne sontelles produites que par nos sens?

- La certitude / le doute : Le doute est-il utile? Qu'est-ce qu'une certitude?

- La vérité : Comment savoir ce qui est vrai ? «Si c'est logique, est-ce nécessairement vrai ?». Faut-il chercher à découvrir la vérité ? La vérité est-elle toujours facile à trouver?

- La fausseté : Comment savoir ce qui est faux? Est-ce qu'on reconnaît ce qui est faux?

-L'apprentissage: Apprendre, est-ce que cela nous transforme? Est-ce qu'on arrête d'apprendre un jour? Peut-on apprendre tout seul? 
-L'intelligence : L'intelligence est-elle le propre de l'humain? Avons-nous la même façon d'être intelligent? L'intelligence peut-elle grandir?

21 L'ensemble de ces travaux de réflexion sur la connaissance humaine va dans le sens du développement d'une attention portée à la raison humaine, dans sa puissance et dans ses faiblesses. C'était l'ambition de Matthew Lipman, professeur de philosophie logique à l'Université de Columbia au moment où il eut l'idée de créer un programme de philosophie pour enfants. Il souhaitant mettre en place un apprentissage de la pensée, et, plus encore, une formation de l'esprit logique et critique. «La vraie rationalité connaît les limites de la logique (...) ; elle sait que l'esprit humain ne saurait être omniscient, que la réalité comporte du mystère. (...) Elle est non seulement critique, mais autocritique" (Morin, 1999b: 22). Ici, les deux penseurs se rejoignent: pour Lipman, les enfants devaient apprendre à manier des habiletés de pensée grâce à la philosophie : il en citait quatre principales et d'autres secondaires. Parmi les habiletés de pensée principales, se trouvait l'habileté d'autocorrection. Il considère que le facilitateur doit inciter les enfants à s'autocorriger, c'est-à-dire à évaluer le sens et la pertinence des énoncés, de façon individuelle et de façon collective. Il s'agit, ainsi, de vérifier la validité des hypothèses, de vérifier leur pertinence, leur sens, et d'évaluer leur sens. C'est cette exigence que j'ai voulu préserver en mettant en lumière la compétence de pensée « critiquer ».

\section{La méthode de la discussion philosophique, propice à l'exercice d'une véritable compréhension intersubjective}

\section{La discussion philosophique, lieu de dialogue intersubjectif}

Bien que l'atelier philosophique soit un lieu privilégié de valorisation de la pensée et de la parole de l'enfant, reconnu comme « interlocuteur valable » (Lévine, 2008), il ne vise pas à simplement recueillir une juxtaposition d'idées individuelles exprimées. Au contraire: son ambition pédagogique est davantage de développer les facultés de dialogue, d'échange et de co-construction d'une pensée collective. Un véritable dialogue se définit, selon Marie-France Daniel (1992) par la création de relations intersubjectives entre les interlocuteurs grâce auxquelles les esprits se décloisonnent pour penser ensemble : la tâche du facilitateur sera de stimuler sa création, par son action pédagogique (que nous détaillerons ci-après). Le déploiement de la recherche philosophique exige l'intersubjectivité - l'interpénétration des esprits et des discours - car c'est la rencontre d'une pluralité d'idées, d'hypothèses, de point de vue qui crée le mouvement de la pensée (Platon, 1994). Ce processus résonne fortement avec les souhaits d'Edgar Morin, qui estime que la compréhension humaine « comporte une connaissance de sujet à sujet (Morin, 1999b: 105). Petit à petit, les enfants accompliront des actes intellectuels qui manifestent l'intersubjectivité: lorsqu'un enfant réagit à l'idée de son camarade, lorsqu'un autre reformule Ainsi, le facilitateur vise la création d'une réflexion co-construite, au fil des interventions, au long d'un travail coopératif (Connac, 2004). La coopération intellectuelle se fonde sur un travail de compréhension mutuelle, dans laquelle les réflexions individuelles se rencontrent et se mêlent. Celle-ci est manifeste lorsque surgissent des moments de dialogisme (Bahktine, 1987), dont la caractéristique est « la présence du discours de l'autre, des 
autres, dans mon propre discours » » (Tozzi, $2002: 282)$. Étant donné qu'il est difficile de mesurer l'intersubjectivité, mais il est fécond d'analyser les discours d'enfants qui, au sein des ateliers philosophiques, livrent des manifestations du dialogisme. Ils se réfèrent aux propos de leurs camarades, ils reprennent leurs concepts, ils explorent leurs idées, ils reformulent les pensées, ils approfondissent les hypothèses de leurs pairs ou expriment un désaccord avec une idée (ainsi que nous le verrons dans la retranscription ci-dessous). Par ces moments de dialogisme, ils nous apportent des indices d'une véritable tentative de compréhension humaine intellectuelle et humaine car ils prennent avec eux les mots d'autrui, embrassent leurs propos, les embarquent dans leur cheminement intellectuel. Nous verrons ci-après les techniques pédagogiques visant le développement de l'intersubjectivité et du dialogisme, ainsi qu'un extrait esquissant une analyse de pratique.

\section{Analyse de gestes spécifiques à l'instauration du dialogue et des mouvements de compréhension mutuelle}

23 Le développement du dialogue repose entièrement sur la facilitation mise en place par l'adulte. Il a à sa disposition plusieurs techniques pour accompagner les enfants dans cette démarche, et les détaillons donc ici.

24 Développer la reformulation : La reformulation est loin d'être un acte intellectuel anodin, car il permet de créer le lien entre deux pensées. Lorsqu'un individu tente de reformuler la pensée d'un autre, il tente de créer un pont afin que les deux idées soient porteuses d'un sens proche. Dans la discussion philosophique, la construction constante de ces ponts permettra aux enfants de passer du temps à explorer leurs idées mais aussi celles de leurs camarades. Pour cela, il est possible de désigner un ou plusieurs reformulateurs parmi les participants, ainsi que le préconise Michel Tozzi, dans la méthode de la Discussion à Visée Démocratique et Philosophique. Ils seront interpelés régulièrement par l'animateur afin de reformuler ce qui vient d'être dit.

25 Inciter à l'entraide : Lorsque les enfants philosophent, ils tentent de formuler leurs idées, qui sont parfois nouvelles ou complexes. Par suite, ils ne parviennent pas toujours à trouver leurs mots, ou à déployer pleinement leur pensée. C'est alors qu'au lieu de mettre l'élève face à un échec, il est porteur de montrer qu'il est normal, parfois, de ne pas y parvenir, et de proposer aux autres participants de venir en aide. En disant, par exemple, "Qu'est-ce qu'il/elle voulait dire à votre avis? ", «Est-ce que vous avez compris ce qu'il/elle essayait de penser?». Les propositions sont en général étonnantes et créent une solidarité dans la recherche.

26 Créer une complémentarité des interventions. Si un enfant propose une idée, il est judicieux de proposer à un autre d'apporter un argument, et un autre de donner un exemple. Puis à d'autres de définir les mots-clés qui étaient présents dans l'énoncé. Le facilitateur incite les participants à construire une complémentarité avec l'idée d'autrui. Ainsi, les enfants apprennent à compléter les idées de leurs camarades, et ainsi, à se décentrer pour réfléchir dans la continuité d'autrui.

27 Stimuler les réactions et les réflexions sur les idées exprimées. Lorsqu'un ou plusieurs enfants expriment une idée qui semble fécondes, le facilitateur peut proposer au groupe de réagir et de prendre le temps d'y réfléchir. Il peut alors répéter l'idée et demander "Qu'en pensez-vous? Souhaitez-vous réagir?». Ces questions anodines engendrent chez les participants à regard réflexif sur la pensée d'autrui. 
28 Développer les prises de position critiques. Dans l'atelier philosophique, les participants seront encouragés à exercer leur esprit critique, à exprimer leurs désaccords, à explorer les différences, grâce aux sollicitations du facilitateur. "Cette idée est-elle juste? Est-elle toujours vraie? Est-ce qu'il y a une faiblesse dans cette idée? ", "Êtes-vous d'accord ou pas d'accord?». Ainsi, les enfants prennent une position critique face à une idée, en l'évaluant, en l'examinant, en cherchant sa validité. Se faisant, ils développent également leur faculté de compréhension et - qui plus est leur faculté à comprendre une idée à laquelle ils s'opposent. Critiquer une idée, c'est également entrer en relation avec elle, car un exercice juste de l'esprit critique exige un travail de compréhension réelle de ce que l'on critique.

29 L'ensemble de ces gestes spécifiques permettent aux enfants d'apprendre à se décentrer afin de comprendre la pensée d'autrui, intellectuellement. Comprendre autrui, c'est tenter de le saisir, pour lui-même, depuis lui-même. C'est cette tentative qui sera visée sans cesse dans le dialogue philosophique. Il s'agit, au fond, d'un modèle d'enseignement de la «compréhension mutuelle entre humains, aussi proches qu'étranger » (Morin, 1999b : 15) par le développement de l'empathie cognitive - cette façon de se mettre à la place d'autrui pour penser avec lui, en prenant soin de lui, dans son altérité. Edgar Morin considère qu'un dialogue repose sur une forme de sympathie (au sens propre d'union des ressentis), et prône une "ouverture subjective (sympathique) à autrui » (Morin, 1999b: 113), en vue de lutter contre l'incompréhension, l'intolérance et l'exclusion. C'est précisément de cela dont il s'agit ici : l'éducation à l'ouverture d'esprit, en tant que faculté cognitive et éthique rendant l'individu capable d'accueillir le monde intellectuel d'autrui (Hawken, 2019), avec sympathie, curiosité et esprit critique.

\section{Analyse d'un dialogue philosophique et des processus de " compréhension humaine intersubjective » (Morin, 1999b : 104)}

Dans l'extrait suivant, qui se situe à 30 minutes du début d'une discussion philosophique sur la joie, on peut voir les manifestations de ces actions dialogiques et intersubjectives. On peut voir que ces élèves de CM2 les mettent en place en étant guidés par la facilitatrice ou de façon spontanée: c'est leur neuvième séance de philosophie (au mois de novembre 20184), si bien qu'ils ont commencé à intérioriser les habitudes que je tentais d'initier. Cette retranscription, loin de présenter un échange philosophique exemplaire, permet néanmoins de révéler les gestes propres à l'intersubjectivité et au dialogisme, au des propos des enfants, ainsi que je l'illustre cidessous en amorce de chacune des interventions significatives.

31 «- [Facilitatrice] : Et là je voudrais savoir, d'après vous, s'il est possible de conquérir la joie, c'est-à-dire de la trouver. Imaginez quelqu'un qui n'a plus de joie en lui ; il a une toute petite boule de joie. Est-ce qu'il y a des façons dans la vie pour trouver la joie ? 12 3 pensez ! et on va faire un tour de parole. Est-ce qu'il y a des façons de décider d'être joyeux, de trouver cette joie ? 123 pensez.... On va faire un tour de parole ; alors comme d'habitude, le but du tour de parole c'est que chacun puisse s'exprimer, mais c'est aussi d'écouter tranquillement chaque personne qui s'exprime parce que on apprend beaucoup de choses comme ça, d'accord?

- [Enfant 1] : Pour moi, on conquérit la joie, c'est quand....

- [Facilitatrice] : Ah, tu as oublié ton idée, on dirait. Comment on peut trouver la joie? 
Est-ce qu'on peut trouver la joie comme ça?

- [Enfant 1] : Il faut être content pour conquérir la joie parce que quand on est triste on peut pas avoir de la joie.

- [Enfant 2, réagit à l'idée d'Enfant 1] : Oui, mais alors là du coup, là tu as peut-être raison mais...

- [Enfant 1] : Il faut être content pour connaître la joie ; mais là il y a un gros problème car si il faut déjà et joyeux pour un joyeux si il faut déjà être content pour trouver la joie, ça veut dire qu'on ne peut pas la trouver ; c'est qu'elle arrive de l'extérieur. Il y a des gens qui pensent comme ça effectivement qu'on ne peut pas la décider c'est soit elle arrive sous elle arrive pas.

- [Enfant 3, réagit à l'idée d'Enfant 2] : Pour moi la joie, pour la trouver, par exemple si tu es pas content ou pas contente tu peux penser à quelque chose d'autre pour être heureux.

- [Facilitatrice, reformule l'idée d'Enfant 3] : Donc on dirait que pour toi, on peut trouver la joie par la pensée, super. [Enfant 4]?

- [Enfant 4, réagit à l'idée d'Enfant 3] : Je suis d'accord avec ce qu' [Enfant 3] a dit ; j'ai pas trop d'idées...

- [Facilitatrice, incite à une réaction intersubjective] : Oui, mais c'est déjà intéressant si tu es d'accord. (...). Quelqu'un souhaite réagir?

- [Enfant 5, s'oppose à l'idée de Enfant 3] : Bah déjà la joie tu ne peux pas la trouver comme ça c'est logique ; mais tu peux pas vraiment la prendre toi-même c'est elle qui vient en toi tu peux pas prendre la joie et la prendre sur toi c'est elle qui doit venir ; c'est elle qui doit venir en toi, comme par exemple quand tu es heureux; tu as le sentiment de joie qui vient, donc c'est comme la tristesse ou d'autres trucs.

- [Enfant 6, complète l'idée d'Enfant 5] : La joie elle vient elle-même...

- [Facilitatrice, reformule l'idée d'Enfant 5] : Ok, elle vient elle-même, elle arrive par hasard, c'est ça? On doit l'attendre?

- [Enfant 7, s'oppose à l'idée d'Enfant 5 et la reformule] : Moi je suis pas d'accord avec Enfant 5, parce que moi je dis c'est nous qui décidons quand la joie elle vient quand par exemple on se rappelant de quelque chose qui est drôle ou en regardant une vidéo drôle, ou juste en se regardant avec son ami, on se tape un fou rire, Se marrer juste, ça nous ramène la joie ; là par exemple, je souris, je sais pas pourquoi j'ai la joie et elle est venue toute seule.

- [Enfant 5, exprime le fait que son idée a été reformulée] : bah voilà, c'est ce que je dis. (...)

- [Enfant 8]: On peut faire une bonne action pour être joyeux

- [Facilitatrice, incite à un développement de l'idée d'Enfant 8] : Ah c'est intéressant cela on n'avait pas encore entendu cette idée. Ça peut nous apporter de la joie de faire une bonne action ? Est-ce que vous pouvez développer l'idée d'Enfant 8 ?

- [Enfant 9, développe l'idée d'Enfant 8] : Ça veut dire : on rend les gens heureux et ça nous rend heureux?

- [Facilitatrice, développe l'idée d'Enfant 9]: Donc, dans ce que tu dis, ce n'est pas forcément quelque chose pour nous-mêmes. En fait dans les exemples de la joie dont on a parlé, c'était quelque chose qu'on obtenait pour nous-mêmes : on nous donne un cadeau, on nous raconte une blague ou des choses comme ça ; là ce serait par rapport à quelqu'un d'autre.

- [Enfant 10, complète l'idée d'Enfant 9] : pour moi la joie on peut l'avoir grâce à nos proches ; par exemple des fois quelqu'un est triste et son ami va venir le consoler pour que lui après il est la pêche; pour qu'il ne soit pas triste ou en colère ; cet ami va un peu 
expliquer les choses du coup pour qu'il se remette en place et qu'après il soit joyeux. (...)

- [Enfant 11, complète l'idée de Enfant 8] : Imaginons une personne qui n'a jamais reçu la joie et bien pour qu'une personne la reçoive, on peut lui donner de l'argent, elle sera contente (rires de quelques-uns) ou alors elle aura la joie ; et si on lui offre un cadeau spécial et ben elle sera aussi contente, par contre euh, c'est un cadeau spécial (rires de quelques-uns)...

- [Enfant 12 : [complète l'idée d'Enfant 7]: Je suis d'accord avec Enfant 7 que... quand par exemple on se regarde fixement on peut rigoler comme ça ; aussi on peut s'inventer des blagues, on peut s'amuser comme des fous, ça nous rend heureux.

- [Enfant 13, développe l'idée d'Enfant 8] : En fait c'est la joie qui nous conquérit si par exemple on est triste et qu'on a pas envie d'être heureux ; par exemple sans faire exprès sur ta télé il y a quelque chose de drôle ou quelqu'un prend une tarte, bah forcément ça te fait rire, tu n'es plus triste ; alors que toi tu veux rester triste, mais là c'est la joie qui vient dire dans ta tête : non, non, non il faut pas que tu sois triste là...

- [Enfant 14, s'oppose l'idée d'Enfant 13] : Bah moi quand tu es triste tu veux la joie mais t'arrives pas à l'avoir, tu essaies de l'avoir mais tu n'y n'arrives pas...

- [Facilitatrice] : C'est intéressant ce que tu dis les autres vous ne l'avez pas encore évoqué on peut se demander si c'est facile de retrouver la joie, quand on est triste par exemple? Est-ce que c'est vraiment si facile?

- [Enfant 14] : Non. Pour moi c'est compliqué.

- [Enfant 3, réagit à l'idée d'Enfant 7] : Pour moi c'est oui et non. Oui, comme tu dis, il y a toujours une part de joie en nous et non parce que quand il y a une plus grosse part de tristesse c'est pas facile; enfin c'est facile et c'est pas facile parce que tu peux repenser à une vidéo ou comme dit Enfant 7 un souvenir et puis tu vas rire; mais tu peux aussi rester sur ta tristesse et pas arriver à te recadrer sur la joie.

- [Enfant 15, réagit à l'idée d'Enfant 5]: Moi je suis d'accord avec Enfant 5 ; par exemple on a la joie, on veut être triste mais on va pas claquer des doigts pour être triste quand même.

- [Facilitatrice, reformule, sous forme de question, l'idée d'Enfant 15] : Et est-ce qu'on peut claquer des doigts pour être joyeux ? Est-ce qu'il suffit de faire " un, deux, trois, joie " et on est joyeux ? Si c'était ça on serait tout le temps joyeux comme des fous en train de rigoler dans la rue ; ça serait bizarre un peu quand même. (Plusieurs enfants claquent des doigts pour essayer d'être joyeux...). J'aurais pas dû faire ça (rires) ; on essaiera à la fin si vous voulez.

- [Enfant 16] : Pour être heureux il faut vouloir il faut la provoquer ; par exemple si il pleut dehors, on peut dire : ah bah non c'est déprimant, il pleut et tu vas dire bah non c'est cool, je vais être mouillée et tout.

- [Enfant 17, reformule l'idée d'Enfant 13] : Je crois que c'est Enfant 13 qui avait dit ça, c'est vrai c'est la joie qui te conquérit, c'est pas à toi de la conquérir.

- [Facilitatrice, synthétise le problème développé par le groupe] : Mais c'est ça le débat, est-ce que c'est nous qui pouvons conquérir la joie, la retrouver la joie, la construire ou bien est-ce que c'est elle qui nous conquiert, elle qui nous trouve ? C'est ça le grand débat dans la vie.»

Cet extrait visait à montrer la multitude des relations intersubjectives manifestes au cours d'un échange philosophique. Nous voyons qu'au fur et à mesure des sollicitations $\mathrm{du}$ facilitateur, qui veille à stimuler les interactions entre les enfants, ces derniers acquièrent un habitus intellectuel majeur : l'habitude de tenter de penser avec autrui, 
de le comprendre pour se mettre en lien avec lui, que ce soit dans l'accord ou le désaccord. Petit à petit, les ateliers philosophiques oeuvrent pour la «rencontre des petits esprits " (Lipman) et mette ainsi en place un processus d'enseignement de la compréhension humaine et de la sympathie intellectuelle.

\section{L'éducation complexe et la formation d'une pensée multidimensionnelle par la pratique philosophique}

Dans un dialogue philosophique avec les enfants, il serait insuffisant de simplement converser et apercevoir le sens. L'exploration approfondie du sens repose sur l'utilisation et le développement des compétences de pensée. Par suite, les recherches ont toujours visé à déterminer l'inventaire des compétences de pensée nécessaire à l'acte de philosopher. Notamment, Matthew Lipman considérait qu'il en existait quatre principales (conceptualiser, raisonner, auto-critiquer et problématiser) et soixante-huit autres secondaires (Lipman, 2008). En France, Michel Tozzi et de nombreux praticiens à sa suite estiment que trois compétences de pensée constituent la didactique du philosopher, auprès des enfants mais aussi sous ses autres formes (conceptualiser, argumenter, problématiser) (Tozzi, 2001). Pour ma part, ayant longuement analysé ces compétences, il m'est apparu que dix compétences de pensée - parmi lesquelles on retrouvera celles que m'ont transmis mes aînés - constituaient de façon essentielle la pratique philosophique : elles sont bien sûr issues des travaux de mes aînés, mais mon expérience, notamment dans la formation d'enseignants ${ }^{5}$, m'a montrée qu'il était nécessaire de trouver un autre modèle. D'une part, je considérais que les trois compétences de pensée tozziennes n'éclairaient pas suffisamment le travail philosophique des enfants, notamment dans sa forme première et fondamentale (commencer par apprendre à questionner, à créer une idée, à illustrer une idée abstraite, et d'autres encore), et se concentraient sur des compétences élaborées et essentielles dans un second temps. D'autre part, les soixante-douze habiletés de pensée décrites par Lipman ne me semblaient pas atteignables dans l'exercice, et, au fond, constituaient une charge pédagogique impossible à assumer. C'est ainsi que, en me nourrissant également de l'analyse des ateliers philosophiques et des compétences qui y étaient utilisées, je suis parvenue à cette typologie unissant ces dix compétences de pensée.

Les compétences de pensée engagent la discussion vers la recherche de complexité caractéristique de la philosophie. Elles permettent d'explorer la pensée dans toutes ses dimensions. Mais surtout, leur efficience dépasse le cadre de l'atelier philosophique: elles sont transversales et opératoires dans la diversité des contextes et des champs de connaissance. Comme le montrent les travaux de Claudine Leleux, la philosophie pour enfants "pourrait jouer ce rôle d'accélérateur dans l'acquisition des compétences cognitives supérieures" (Leleux, 2014: 222-223). Selon l'enseignante belge, elle permettrait d'éviter l'attentisme, au sens où la pratique philosophique serait le cadre méthodologique idéal pour intervenir dans l'éducation de la pensée. In fine, la formation d'une pensée multidimensionnelle, applicable partout, permettrait à l'enfant de clarifier et d'organiser l'appréhension du monde complexe, global, multidimensionnel.

Ces deux aspects, constituant un programme d'apprentissage de la pensée, permettent de répondre à l'appel de la pensée complexe. Afin de rendre les élèves capables 
d'affronter la complexité du monde, il convient de développer certaines compétences de pensée : celles-ci, pratiquées durant la philosophie, pourront être transposées dans les autres domaines du savoir et de l'existence.

\section{La formation d'une pensée multidimensionnelle par la pratique des compétences de pensée}

L'atelier philosophique aura pour viser de solliciter chez les enfants l'utilisation des compétences de pensée : tout au long du dialogue, progressivement, le facilitateur leur demandera de créer une idée, de poser une question, de trouver un argument, de trouver un exemple, de distinguer deux mots ou deux idées, de raisonner en trouvant la cause ou la conséquence d'une idée. Ainsi, la discussion philosophique permet de jongler alternativement avec ces divers mouvements de la pensée. En outre, à certains moments clés, le facilitateur nommera la compétence de pensée utilisée : soit en la travaillant tout au long de la séance (en mettant en valeur, par exemple, tout au long d'un atelier, chaque argument en verbalisant le fait qu'il s'agit d'un argument), soit en les nommant de façon régulière afin de transmettre petit à petit leur connaissance aux enfants. De cette façon, la verbalisation des compétences de pensée engendre une conscientisation de la part des enfants : devenant conscient de leur pensée et des outils dont elle dispose, il aura ensuite le pouvoir de les utiliser à leur gré. Cela sera bien entendu déclaré aussi par le facilitateur, qui valorisera leurs compétences et les engagera à s'en servir au quotidien.

En un sens, les compétences de la pensée philosophique permettent de réaliser le modèle de la pensée complexe (Morin, 1990), dans la mesure où il s'agit d'appréhender une question philosophique au moyen de divers angles de la pensée. L'utilisation de chaque compétence éclaire différemment la texture du problème. Mais surtout, l'atelier philosophique constitue un tremplin de choix pour opérer les boucles dialogiques que préconisent Edgar Morin (Morin, 1982) : boucles entre philosophie et sciences, boucles interdisciplinaires et transdisciplinaires. En posant une question philosophique universelle, les apprentis penseurs mobiliseront inéluctablement des savoirs issus d'autres champs de connaissance (qui seront, aussi, interrogés lors d'un temps dédié, extérieur à l'atelier). La complexité du problème pourra alors être aperçue, car la discussion philosophique l'aura éclairé sous un angle conceptuel, ainsi que sous un angle scientifique, historique, sociologique.

\section{Une analyse des compétences multidimensionnelles et de leur apport pour l'appréhension du monde complexe.}

38 Il convient d'analyser les principales compétences de pensée mobilisées en philosophie, afin de saisir leur portée au sein du paradigme de l'éducation complexe. Elles sont au nombre de $\mathrm{dix}^{6}$, mais certaines méritent une analyse plus précise (je laisse sciemment de côté, les compétences d'illustration et de raisonnement) :

39 Questionner : Afin de ne pas subir la réalité du monde et de la société contemporaine, il convient de transmettre l'importance du questionnement, par lequel chacun s'autorise (re)mettre en question le monde, dans sa globalité et dans son actualité.

40 Créer une idée. Face aux questionnements, chacun sera légitime pour rechercher une idée qu'il considère sienne, soit en la créant («je pense que... ») soit en se l'appropriant. 
La philosophie appelle le développement d'une pensée créative, afin de ne pas rester soumise à la réalité effective. La créativité permet d'imaginer des hypothèses nonréalistes, extérieures au monde actuel. Edgard Morin met en garde contre les ethnocentrismes, sociocentrismes et les problèmes de hauteur de vue. En se dégageant des contextes, déterminismes et cultures dominantes, la créativité philosophique permet de penser le monde autrement, et de prendre conscience qu'il pourrait être différent.

41 Conceptualiser. L'un des éléments faisant de la complexité du monde une opacité réside dans l'utilisation non-interrogée de termes conceptuels. Liberté, bonheur, amour, travail, individu, société, différence, égalité, justice, existence, vie, mort, vérité, illusion, et d'autres encore. Ainsi, les ateliers philosophiques dédient un temps conséquent à leur conceptualisation, afin que les élèves, au fur et à mesure de leur réflexion, acquiert un regard plus clair sur le sens.

42 Argumenter. L'exigence d'argumentation dans la réflexion philosophique permet d'engager les élèves à prendre l'habitude de rechercher des justifications, des preuves, des arguments, afin de vérifier la validité d'une idée. La compétence à argumenter joue donc un rôle épistémologique fort, en vue de cibler les erreurs, préjugés et aveuglements.

43 Problématiser. Une problématique philosophique révèle une tension, un nœud, une difficulté tant dans le monde des idées que dans le monde réel. Elle cible un nœud de la condition humaine, et l'éclaire ainsi dans sa complexité. En effet, elle surgit, en général, de l'articulation de deux ou plusieurs idées, théories, expériences dont la coexistence réelle semble paradoxale ou même contradictoire. Développer la compétence de problématisation, c'est donc apprendre à voir l'endroit où le monde est complexe.

44 Distinguer. Il est essentiel de parvenir à organiser le sens et les connaissances. Dans la continuité de la conceptualisation, la faculté de distinguer les mots, concepts, et idées permet de clarifier leurs significations, dans leurs ressemblances mais aussi leur différence. La distinction est fondamentale pour avancer dans la pensée sans confusion, et enseigner une attention aux nuances existant dans les mots et les réalités.

45 Critiquer : L'exercice de l'esprit critique est fondamental dans l'atelier philosophique, dans la mesure où il transmet une éducation au doute. L'autocritique permet de débusquer autant les erreurs du groupe que les siennes propres: stimulée par le facilitateur, elle constitue des temps de pause et de distanciation vis-à-vis du contenu produit par le groupe, afin de vérifier sa validité. La critique permet notamment de s'interroger à propos de nos présupposés, de nos opinions non-interrogées, de nos oublis, de notre contexte. On voit ici qu'elle réalise un travail de recherche vis-à-vis des différents types d'erreurs qui peuvent encombrer la pensée : si, comme le montre Edgar Morin, nos idées sont le produit d'un aveuglement, d'un déterminisme culturel, d'une idéologie, il est possible que l'examen critique collectif les débusque.

46 Dialoguer. Il peut sembler étonnant de considérer le dialogue comme un acte de la pensée. Pourtant, lorsqu'on pense, intérieurement, en prenant en compte l'idée d'autrui - en y réagissant, en la reformulant, en s'y opposant, on est bien en train de penser avec autrui. Chaque fois que notre processus de pensée intègre des éléments issus de la pensée d'autrui, on exerce une pensée dialogique. Le dialogue est donc une compétence de pensée centrale dans la recherche philosophique, au sens où elle nous permet de déplacer notre pensée individuelle au moyen d'une pensée divergente. Cette démarche intellectuelle contient, selon Claudine Leleux, une qualité citoyenne, dans la 
mesure où elle correspondant à un développement de l'ouverture d'esprit par le « vécu pluraliste » éprouvé dans l'écoute et la confrontation à autrui (Leleux, 2014 : 187-188).

47 Ayant intériorisé les compétences de pensée (qui sont d'ores et déjà présentes à l'état potentiel dans la cognition de tous), les enfants et adolescents se sentiront capables de les mobiliser dans tous les contextes : à l'école, à la maison, face aux informations, aux divers énoncés qu'ils entendent, lisent et reçoivent. Ils auront pris connaissance de ces multiples outils intellectuels dont peuvent devenir maîtres. Or ces compétences permettent de construire un regard plus clairvoyant sur le monde et sa complexité. En somme, une pratique de la pensée, fréquente et consciente, permet à l'enfant de s'approprier cette faculté dans toutes ses dimensions et d'en faire une capacité stable et fiable, permanente et constante face au monde complexe, changeant et multidimensionnel.

\section{Conclusion}

48 Le paradigme de l'éducation complexe pose de multiples exigences afin de redonner du sens aux apprentissages, au rapport au savoir, et, surtout, au rapport au monde. Il est possible que la crise éducative soit née d'un oubli : celui du lien - entre les savoirs, entre les individus, entre les dimensions de la pensée. C'est en tissant à nouveau ces liens que les élèves pourront apercevoir la complexité des choses. Paradoxalement, c'est l'étude de la complexité qui pourrait leur apporter davantage de clarté dans leur vision de l'existence.

49 Ainsi, la pratique de la philosophie avec les enfants et adolescents s'inscrit dans la volonté de tisser à nouveau le sens dans toute sa complexité. D'abord, en dédiant un temps scolaire à la réflexion sur la condition humaine, dans ses toutes ses dimensions, notamment éthique et épistémologiques: en faire l'économie, c'est condamner les élèves à ne pas comprendre le contexte, la texture des savoirs, des sociétés et des connaissances qu'ils acquièrent.

50 Outre une meilleure compréhension du monde, dans toute sa complexité et son actualité, la pratique du dialogue philosophique permet de mieux comprendre autrui, en un sens fort. Non pas comprendre au sens de recevoir une signification, mais comprendre au sens de prendre l'autre avec soi, d'observer la pensée d'autrui, de chercher son sens depuis l'autre. Autour de grandes questions unissant les intérêts des élèves, ils ont l'occasion d'échanger leurs idées, dans toute leur pluralité. À l'échelle micro-pédagogique, l'atelier philosophique rejoue ce qui se joue à l'échelle macrosociétale : la faculté d'accéder véritablement à autrui ne surgit pas dans la spontanéité des histoires et cultures complexes, elle s'enseigner grâce à l'action volontaire d'un porteur et de ses interlocuteurs.

51 Ce parcours est d'autant plus porteur que les élèves sont unis par un cheminement collectif de la réflexion, où chacun mobilise ses compétences de pensée : l'utilisation des compétences de pensée multidimensionnelles, dont l'efficience réside dans l'exploration approfondie des premières idées.

52 La philosophie pour enfants réalise un objectif fort du paradigme de la pensée complexe : étudier la condition humaine, tout en découvrant non la nécessité de la rencontre intersubjective pour en saisir son-ses sens, mais aussi le besoin de l'analyser en utilisant toutes les compétences potentielles de la pensée humaine. 


\section{BIBLIOGRAPHIE}

Bakhtine, M. (1987), Esthétique et théorie du roman, Paris : Gallimard Tel.

Chirouter, E. (2007), Lire, réfléchir et débattre à l'école élémentaire : La littérature de jeunesse pour aborder des questions philosophiques. Paris : Hachette.

Connac, S. (2004), Discussions à visée philosophique et classes coopératives en ZEP. Doctorat, Montpellier 3.

Daniel, M.-F. (1992), La philosophie et les enfants. Les modèles de Lipman et de Dewey. Montréal : Les Éditions logiques, 1992.

Descartes, R. (1996), Principes de la philosophie, in A. Charles \& P. Tannery, Euvres complètes. Paris : Librairie philosophique Vrin.

Hawken, J. (2019), 1, 2, 3, Pensez! Philosophons les enfants. 10 règles d'or et outils pédagogiques. Lyon : Chronique Sociale.

Leleux, C. (2005), (dir.), La philosophie pour enfants. Le modèle de Matthew Lipman en discussion. Bruxelles : De Boeck.

Leleux, C. (2014), « Apprendre à penser dès 5 ans à l'épreuve du modèle de Matthew Lipman ?", in C. Leleux (dir.), La philosophie pour enfants. Le modèle de Matthew Lipman en discussion. Paris : Librairie Vrin.

Lévine, J. (2008), L'enfant philosophe, avenir de l'humanité ?. Paris : ESF éditeurs.

Lipman, M., Oscanyan, F.S., Sharp, A.-M. (1990), Philosophy in the classroom. Philadelphie : Temple University Press, $2^{\text {nd }}$ edition.

Lipman, M. (2008), À l'école de la pensée, trad. N. Decostre. Bruxelles : De Boeck, 2 ème édition.

Morin, E. (1982), Science avec conscience. Paris : Éditions Points.

Morin, E. (1990), Introduction à la pensée complexe. Paris : Éditions Points.

Morin, E. (1999a), La tête bien faite. Repenser la réforme, réformer la pensée. Paris : Édition du Seuil.

Morin, E. (1999b), Les sept savoirs nécessaires à l'éducation du futur. Paris : Éditions du Seuil.

Pettier, J. C. (2004), Apprendre à philosopher. Lyon : Chronique Sociale.

Platon (1994), Théétète, trad. M. Nancy. Paris : Garnier Flammarion.

Sasseville, M. (2009), La pratique de la philosophie avec les enfants. Laval : Les Presses de l'Université Laval.

Tozzi, M. (2001) (dir.), L'éveil de la pensée réflexive à l'école primaire. Paris : Hachette Education. Tozzi, M. (2002), (dir.), Nouvelles pratiques philosophiques en classe, enjeux et démarches. Rennes : Cndp-Crdp de Bretagne.

Tozzi, M. (2007), La philosophie : état des lieux-Rapport de l'UNESCO. La philosophie ou l'école de la liberté. Paris : Éditions UNESCO. 


\section{NOTES}

1. Edgar Morin fait partie notamment du Conseil d'Orientation de la Fondation SEVE, qui organise des formations pour devenir animateurs d'ateliers de philosophie pour les enfants.

2. Selon Edwige Chirouter, la littérature jeunesse serait un tremplin fécond pour la pensée philosophique, dans la mesure où elle permet d'aborder les questions et concepts au travers de personnages qui les incarnent, s'y confrontent et permettent donc de les problématiser.

3. Extraits d'un atelier philosophique, Temps méridien, école G. Péri (Romainville, Seine SaintDenis), 18 janvier 2013.

4. L'extrait est issu d'une séance de philosophie au sein du Dispositif "Classe Idée » : 16 novembre 2018, à l'école élémentaire Fraternité (93 230 Romainville)

5. Dans le cadre de l'Académie de Créteil et d'un projet porté avec la Circonscription académique Les Lilas/Romainville/Pré Saint-Gervais, mais aussi dans le cadre de l'Académie de Versailles, au sein du Projet PhiloJeunes.

6. Pour rappel, voici la liste: questionner, créer une idée, conceptualiser, argumenter, problématiser, illustrer, distinguer, raisonner, critiquer, dialoguer.

\section{RÉSUMÉS}

En vue de constituer des pratiques pédagogiques pertinentes pour l'élaboration d'une éducation complexe, il convient de prendre en compte un mouvement de la Philosophie Pour Enfants (PPE), tel qu'il se développe dans le monde depuis les années 1970. En effet, la pratique de la philosophie entre cinq et dix-sept ans vise des objectifs résonnant fortement avec les travaux d'Edgar Morin : tant dans l'objet d'étude (la condition humaine, dans sa forme expérientielle, accessible à tous dès le plus jeune âge), que dans sa méthode (la discussion philosophique travaillant la compréhension humaine et intersubjective, animée par l'utilisation de compétences de pensée multidimensionnelles). L'atelier philosophique pourrait être un tremplin pour accompagner les élèves dans la découverte de la texture complexe du monde et des idées.

In order to create pedagogical practices that are pertinent for the development of a complex education, on should take into account the Philosophy for Children (P4C) movement, as it has been flourishing in the world since the seventies. Indeed, the practice of philosophy with children from 5 to 17 aims at goals that are very close to the research work of Edgar Morin: regarding its subject matter (the human condition, in its experimental form, accessible to all from a young age), but also its methods (the philosophical discussion, aiming for the exploration of human and intersubjective understanding, through the use of muldimensional thinking skills). The philosophical workshop could be a significant and fruitful method to help students to discover the complex structure of ideas and realities. 
INDEX

Mots-clés : philosophie, condition humaine, dialogue, éthique, épistémologie, intersubjectivité, compétence de pensée

Keywords : philosophy, human condition, dialogue, ethics, epistemology, intersubjectivity, thinking skill

\section{AUTEUR}

JOHANNA HAWKEN

Université Picardie Jules Verne 